\title{
Bestimmung des Schneevorrates in Einzugsgebieten mittels Fernerkundungsmethoden
}

Zusammenfassung: Im vorliegenden Beitrag werden der aktuelle Stand von satellitenbasierten Methoden zur Bestimmung des Schneevorrates in Einzugsgebieten für österreichische Verhältnisse dargestellt und die jeweiligen Stärken und Schwächen für wasserwirtschaftliche Anwendungen diskutiert. Anhand zweier Fallbeispiele der Validierung eines flächendetaillierten Schneemodells und der regionalen Wasserbilanzmodellierung - wird gezeigt, dass Fernerkundungsmethoden in Verbindung mit hydrologischen Simulationen eine wesentliche Verbesserung der Bestimmung des Schneevorrates gegenüber den konventionellen Punktmessungen ermöglichen.

Using methods of remote sensing for determining snow reserves

Summary: This paper presents the current state of the art of satellite-based methods for estimating snow cover characteristics in Austrian catchments and discusses their pros and cons for water resources applications. Based on two case studies - validating a spatially distributed snow model and regional water balance modelling the paper illustrates that the remote sensing methods can significantly improve the estimates of catchment snow packs as compared to traditional point measurements, when combined with hydrological simulations.

\section{Einleitung}

Der Schnee als Teil der Wasserbilanz spielt in der Wasserwirtschaft in mehrerer Hinsicht eine wichtige Rolle. In vielen Gebieten Österreichs liefert der in Form von Schnee gespeicherte Winterniederschlag einen wichtigen Beitrag zum Wasserdargebot im Frühjahr, das unter anderem für Wasserversorgung und Wasserkraft genutzt wird. Umgekehrt kann der als Schnee im Gebiet gespeicherte Niederschlag wesentlich zu Hochwässern beitragen (Resz-

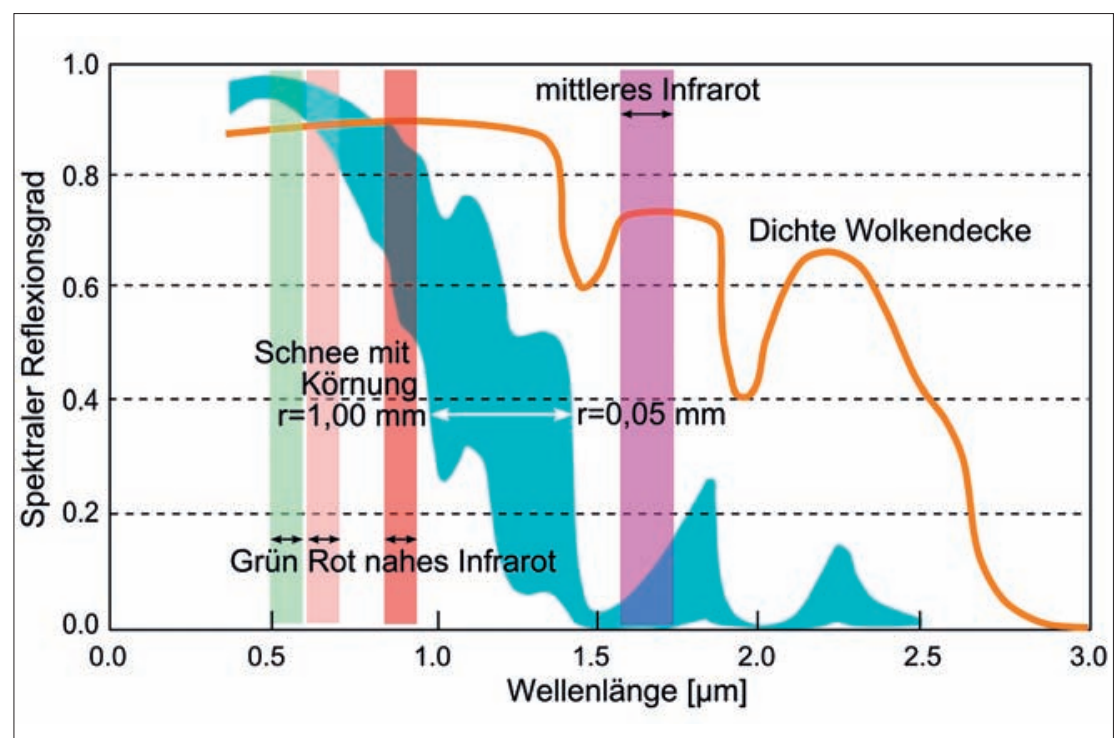

Abb. 1: Spektraler Reflexionsgrad von Schnee (hellblaue Fläche) und Wolken (rote Linie) (adaptiert von Dozier 1989).

ler et al. 2008). Eine genaue Kenntnis des Schneerückhalts ist für die Planung und Bewirtschaftung wasserwirtschaftlicher Systeme wichtig. Während traditionell der Schneevorrat über Begehungen im Gebiet (Schneekurse) bestimmt wurde, sind in den letzten Jahrzehnten Fernerkundungsmethoden hinzugekommen, die es ermöglichen, den Schneevorrat flächendeckend $\mathrm{zu}$ bestimmen. Flächendeckende Aussagen sind sowohl für die Abschätzung des gesamten Schneevorrates in einem Gebiet als auch zur Bestimmung der räumlichen Verteilung in Teilgebieten sehr interessant.

Die Schneebeobachtung in der Fernerkundung konzentriert sich im Wesentlichen auf zwei Kenngrößen der Schneedecke: Die Schneebedeckung und die Veränderung der Feuchte. Die Schneebedeckung kann direkt aus Aufnahmen der optischen Fernerkundung bestimmt werden, die Veränderung der Schneefeuchte aus Mikrowellen-Aufnahmen mittels Synthetic Aperture Radar (SAR). Theoretisch könnte man noch weitere Eigenschaften ableiten, wie Körnung, Verschmutzungsgrad, Temperatur der Oberfläche, Schnee- höhe, etc. Allerdings bedarf es dann eines mente und weniger zuverlässiger Methoden, sodass in der Praxis diese Parameter nicht abgeleitet werden. Neben den Eigenschaften der Schneedecke sind für die Bestimmung des Schneevorrates noch einige weitere Daten von Bedeutung, welche man ebenfalls aus Fernerkundungsaufnahmen erhält: das digitale Geländemodell und die davon ableitbaren Größen (wie z. B. Hangneigung und -exposition, Sonneneinstrahlung, u.a.).

In der vorliegenden Arbeit werden die aktuellen Möglichkeiten der Fernerkundung zur Bestimmung der Schneeverhältnisse in Einzugsgebieten diskutiert. Besonderes Augenmerk wird auf aktuelle Sensoren gelegt, insbesondere MODIS, da dieser eine sehr interessante Datenquelle aus wasserwirtschaftlicher Sicht darstellt. An hand zweier Fallbeispiele wird gezeigt, in welcher Weise Fernerkundungsinformation zielführend für wasserwirtschaftliche Aufgabenstellungen herangezogen werden kann, wobei der Kombination mit hydrologischen Modellen ein hoher Stellenwert zukommt. größeren Aufwandes, spezieller Instru- 


\section{Fernerkundung von} Schneekenngrößen

Die optische Fernerkundung nützt die von den Objekten reflektiere Sonnenstrahlung in den Wellenlängenbereichen vom nahen Ultraviolett bis zum mittleren Infrarot. Vom Weltraum aus ist der Einsatz von multispektralen Sensoren heute Standard. Typische Vertreter im mittleren bis groben räumlichen Auflösungsbereich sind Landsat Thematic Mapper (Landsat 2009), Systeme auf den SPOT Satelliten (Spot 2009), AVHRR sowie MODIS (MODIS 2009). Kenngrößen dreier Sensoren sind in Tabelle 1 zusammengefasst. Während SPOT durch seine relativ hohe räumliche Auflösung punktet, ist MODIS aufgrund seiner hohen zeitlichen und spektralen Auflösung von Interesse.

Abbildung 1 zeigt typische spektrale Kurven für Schnee in Abhängigkeit von der Korngröße. Weitere Abhängigkeiten im Reflexionsgrad sind durch den Verschmutzungsgrad gegeben. Schnee unterscheidet sich im Allgemeinen sehr deutlich von seiner Umgebung und deshalb beschränkt man sich sinnvollerweise auf die Feststellung der Ausdehnung der Schneedecke. Da Schnee im sichtbaren Wellenlängenbereich, ähnlich den Wolken, einen hohen Reflexionsgrad hat, wäre eine Schneeklassifizierung in Bildern, in denen auch Wolken auftreten, kaum durchführbar. Verwendet man den mittleren Infrarot-Kanal dazu, wie ihn z.B. der SPOT Sensor oder MODIS anbietet, wird eine Unterscheidung möglich. Abbildung 1 zeigt sehr deutlich die verschiedenen Reflexionseigenschaften von Wolken und Schnee, je weiter man sich vom sichtbaren Spektrum in Richtung mittleres Infrarot entfernt.

Alle optischen Sensoren sind auf Tageslicht und auf wolkenfreie Bedingungen angewiesen. Bei aktiven Mikrowellen-Sensoren hingegen ist beides nicht der Fall. SAR (Synthetic Aperture Radar)-Systeme stellen ihre eigene „Beleuchtung“ zur Verfügung und können die Wolkendecke fast ungehindert durchdringen. Mehrere Satelliten sind mit einem solchen System ausgestattet, wie etwa ERS2-AMI und ENVISAT-ASAR, die im C-Band arbeiten (4-8 cm Wellenlänge). Beide Satelliten werden durch die European Space Agency betrieben (ESA 2009). Allerdings führt der steile Blickwinkel im Gebirge $\mathrm{zu}$ sehr störenden Effekten, und Speckles (eine rauschähnliche, durch Interferenz hervorgerufene Störung) reduzieren die Zuverlässigkeit der Interpretation. Das im Mikrowellenbereich empfangene Signal

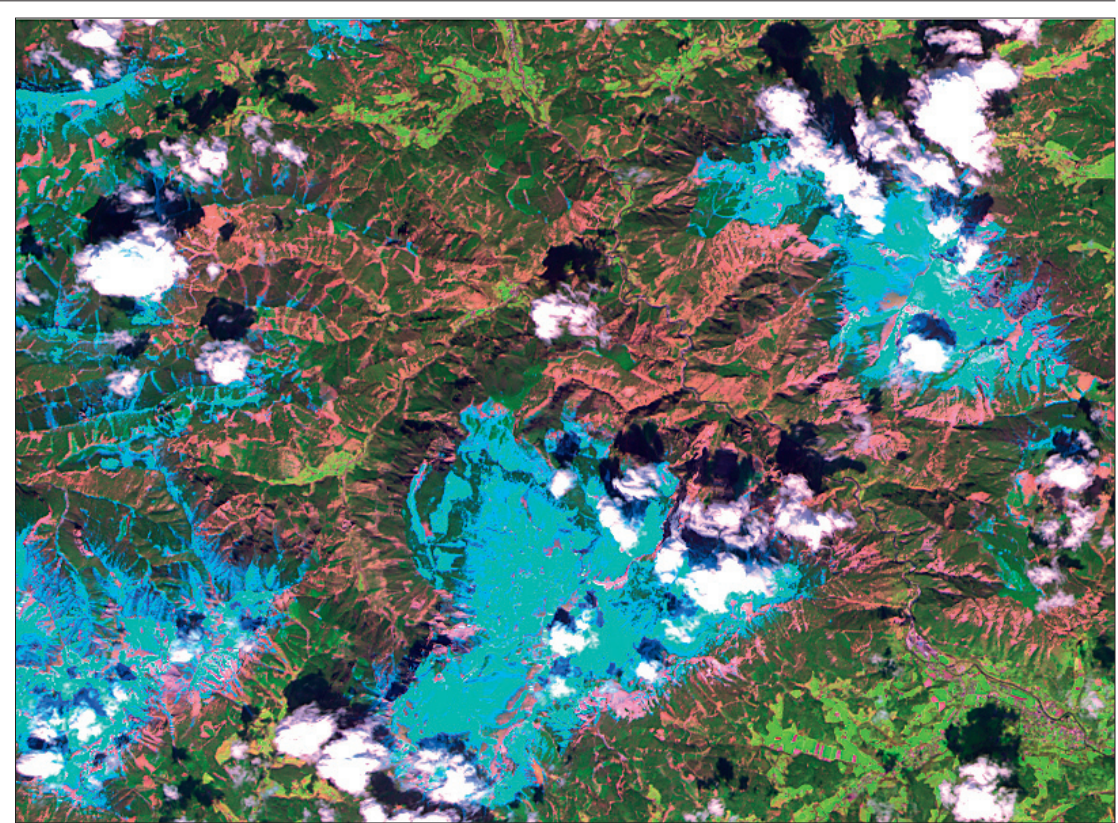

Abb. 2: Schneeverhältnisse im Gebiet Schneealpe-Rax-Schneeberg am 28. April 2004. SPOT Satellitenbild mit mittlerem Infrarot-Kanal (links). Schneeklassifikation (rechts): Dunkelbraun = kein Schnee, Cyan = volle Schneedecke, Gelb = Wolken, Rot = unzuverlässiges Klassifizierungsergebnis (hier Wolkenschatten).

\begin{tabular}{|c|c|c|c|}
\hline Sensor & $\begin{array}{l}\text { Advanced Very High } \\
\text { Resolution Radio- } \\
\text { meter (AVHRR) }\end{array}$ & $\begin{array}{l}\text { Moderate Resoluti- } \\
\text { on Imaging Spec- } \\
\text { troradiometer } \\
\text { (MODIS) }\end{array}$ & $\begin{array}{l}\text { Haute Résolution } \\
\text { dans le Visible et } \\
\text { I'Infra-Rouge } \\
\text { (HRVIR) }\end{array}$ \\
\hline Satellit & $\begin{array}{l}\text { verschiedene NOAA } \\
\text { Satelliten }\end{array}$ & EOS Terra und Aqua & SPOT 4 bzw. 5 \\
\hline Beginn & $\begin{array}{l}\text { je nach Sensor, z.B. } \\
\text { AVHRR/3: } 1998\end{array}$ & 2000 & 1998 bzw. 2002 \\
\hline Räumliche Auflösung & ca. $1 \mathrm{~km}$ & ca. $0,5 \mathrm{~km}$ & $20 \mathrm{~m}$ multispektral \\
\hline Zeitliche Auflösung & 1-10 Bilder pro Tag & 1-2 Bilder pro Tag & $\begin{array}{l}\text { In Österr. theoretisch } \\
\text { ca. } 3 \text { Tage bei Nutzung } \\
\text { beider Satelliten und } \\
\text { programmierter } \\
\text { Sensorneigung }\end{array}$ \\
\hline Wellenlänge & Sichtbar-Infrarot & Sichtbar-Infrarot & Sichtbar-Infrarot \\
\hline Probleme & Wolken & Wolken & Wolken \\
\hline $\begin{array}{l}\text { Termingerechte Ver- } \\
\text { fügbarkeit }\end{array}$ & $<1 \mathrm{~h}$, wenn registriert & ca. 2 Tage & $\begin{array}{l}\text { Nach erfolgreicher } \\
\text { Aufnahme ca. } 3 \text { Tage }\end{array}$ \\
\hline Kosten & Auf Anfrage & keine & $\begin{array}{l}\text { Abh. von Prioritätsstufe } \\
\text { ca. EUR } 3000\end{array}$ \\
\hline url & http://www.noaa.gov & http://www.nsidc.org & $\begin{array}{l}\text { http://www.spotimage. } \\
\text { fr }\end{array}$ \\
\hline
\end{tabular}

des Schnees resultiert aus einer Summe von Einflüssen: die Dielektrizitätseigenschaften, die Temperatur, Dichte, Alter und Struktur. Bei dem C-Band $(5,3 \mathrm{GHz}$ entsprechend $5,7 \mathrm{~cm}$ Wellenlänge), welches bei ERS und ENVISAT Verwendung findet, ist trockener Schnee fast transparent (Ulaby et al. 1986). Die Auswertung der Schneebedeckung und der Schnee-Eigenschaften aus einer einzel- nen SAR-Aufnahme der beiden angeführten Satelliten ist daher kaum möglich. Ein Vergleich einer Referenzaufnahme mit trockenem Schnee und Aufnahmen zu anderen Zeitpunkten, erlaubt aber das Monitoring der Schneeschmelze (Blöschl et al 2002; Nagler und Rott 2000). Passive Mikrowellensensoren (die die Mikrowellenausstrahlung der Erdoberfläche nutzen) kön- 

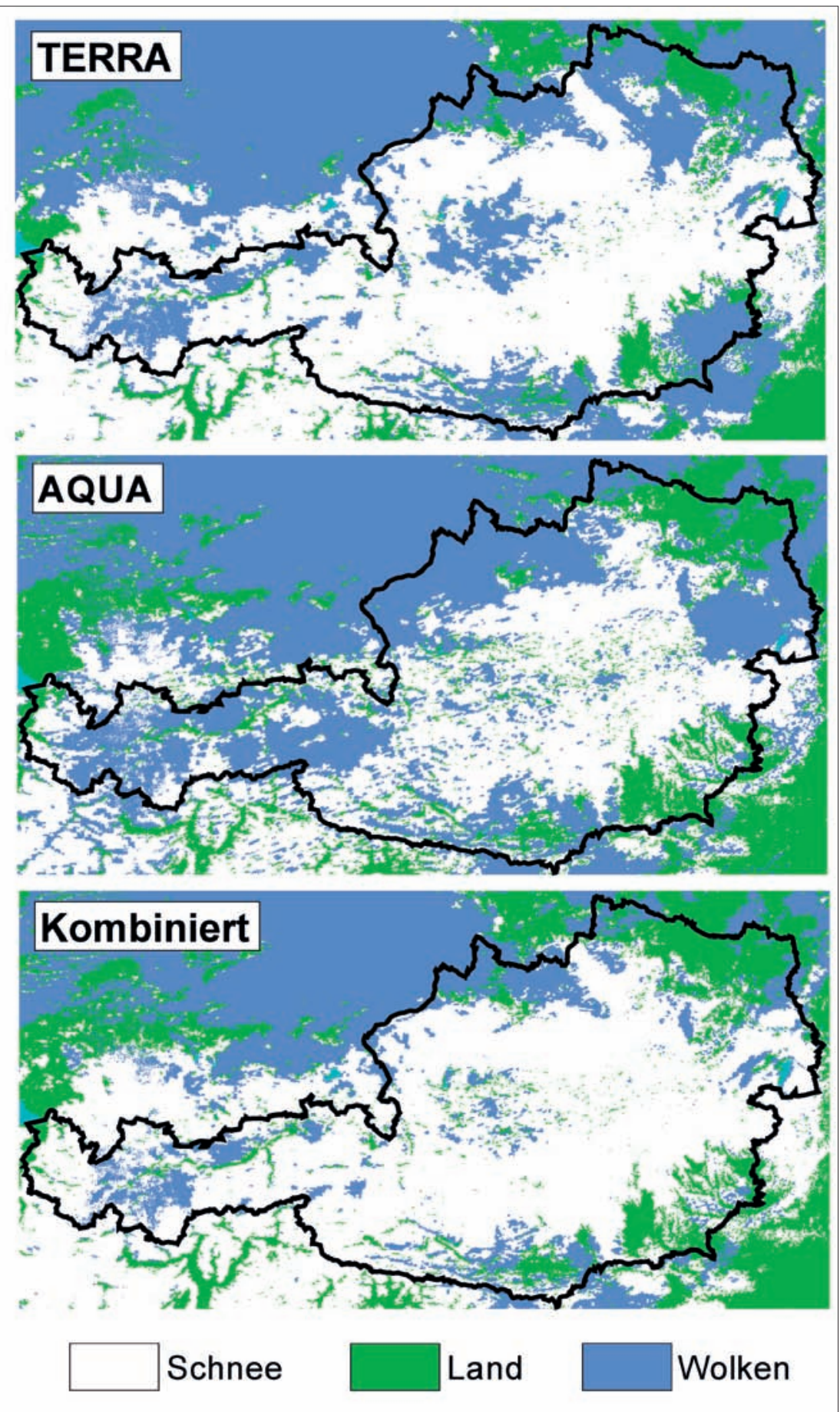

Abb. 3: Beispiel für die Kombination von MODIS Schneebedeckung der beiden Satelliten Terra and Aqua für den 25. Oktober 2003.

nen bei geringmächtigen Schneedecken auch Information über den Schneewasserwert geben (Derksen et al. 2005), allerdings sind die Rasterweiten so groß (>25 km), dass sie für österreichische Verhältnisse nicht sehr aussagekräftig sind. Für praktische wasserwirtschaftliche Zwecke sind deshalb die optischen Sensoren von größter Bedeutung. es sich um ein Maß der Differenz zwischen der empfangenen Strahlung im spektralen Rot-Bereich, wo Schnee sehr stark reflektiert, und jener im spektralen mittleren Infrarot-Bereich bei etwa $1,5 \mu$ m oder $2,0 \mu \mathrm{m}$, wo Schnee sehr schwach reflektiert. Die Differenz wird normiert auf die Summe dieser Strahlung, wodurch das Ergebnis zwischen -1 und +1 zu liegen kommt. Je näher der Wert bei +1 liegt, desto wahrscheinlicher liegt Schnee vor (vergl. $A b b .1$ ). Durch die Normalisierung werden auch Beleuchtungseffekte (das sind zu einem hohen Maße multiplikative Störgrößen) stark reduziert. Mit Hilfe einfacher Schrankenbildung kann man bis zu 4 Klassen recht zuverlässig unterscheiden: kein Schnee, stark lückige Schneedecke, leich lückige Schneedecke, geschlossenen Schneedecke. Die Zuverlässigkeit kann sogar noch etwas erhöht werden, wenn man auch den NDVI (Normalised Difference Vegetation Index) in die Interpretation hineinnimmt. Abbildung 2 zeigt auf der linken Seite ein SPOT4 Satellitenbild im Bereich Schneealpe-Rax-Schneeberg in einer Farbkomposition mit dem mittleren Infrarotkanal. Man sieht, dass Schnee und Wolken deutlich unterscheidbar sind Rechts ist das Ergebnis einer Klassifizierung. Die Klassen „kein Schnee“, „stark lückige Schneedecke“, „leicht lückige Schneedecke“ und „geschlossene Schneedecke" sind in den Farben dunkelbraun, hellbraun, weiß und cyan wiedergegeben Wolken sind in Gelb visualisiert. Die roten Bereiche deuten an, dass dort das Klassifizierungsergebnis nur sehr unzuverlässig bestimmt werden konnte. In diesem Fall waren dafür die Wolkenschatten verantwortlich. Ähnliche Algorithmen auf Basis der Helligkeiten im infraroten und sichtbaren Bereich finden auch bei anderen optischen Sensoren wie etwa MODIS Anwendung (Hall et al. 2001).

Wie erwähnt sind optische Sensoren auf wolkenfreie Bedingungen angewiesen. In Österreich ist die durchschnittliche Wolkenbedeckung etwa $70 \%$. Das bedeutet, dass nur für $30 \%$ der Fläche bzw. Tage mit dieser Art von Sensoren Schneeinformation zur Verfügung steht. Der Anteil der durch Wolken verdeckten Pixel kann jedoch durch geeignete Methoden deutlich verringert werden, wenn Satellitenaufnahmen mit hoher zeitlicher Auflösung zur Verfügung stehen. Eine derartige Methode wurde von Parajka und Blöschl (2008a) entwickelt. Dabei werden vorerst die Bilder unterschiedlicher Satellitenplattformen (in diesem Fall Aqua und Terra) am gleichen Tag kombiniert. Ein Beispiel zeigt Abbil- 
dung 3. Die Schneeklassifizierungen von Terra (oben) und Aqua (Mitte) werden in ein Bild (unten) zusammengeführt, wodurch an diesem Tag die Wolkenbedeckung von $56 \%$ (Terra) bzw. $61 \%$ (Aqua) auf $47 \%$ reduziert werden kann. Eine zusätzliche Reduktion der Wolken ist möglich durch Kombination mit den Bildern der vorhergehenden Tage sowie durch eine Kombination der Pixelwerte mit benachbarten Pixeln. Parajka und Blöschl $(2006,2008 a)$ testeten die Genauigkeit dieser Methode durch einen Vergleich mit Bodenmessungen der Schneehöhe an 754 Stationen in Österreich (Abb. 4). Die Genauigkeit ist hier definiert als Verhältnis der korrekt identifizierten Stationstage zur Gesamtanzahl der Stationstage. Eine Genauigkeit von 90 \% bedeutet beispielsweise, dass im Durchschnitt in Österreich an $90 \%$ der Tage die Schneebedeckung (schneebedeckt bzw. aper) durch MODIS korrekt identifiziert wurde. Die Symbole zeigen verschiedene Methoden zur Reduktion der Wolkenbedeckung jeweils für die Monate Januar und Oktober. Im Januar ist die Genauigkeit der Originaldaten (Aqua bzw. Terra) 89 bzw. $87 \%$. Dabei ist die Wolkenverdeckung knappe $70 \%$. Durch die verschiedenen Methoden kann die Wolkenverdeckung auf $5 \%$ reduziert werden. Allerdings sinkt die Genauigkeit etwas ab (auf 84\%). Im Oktober liegt die Genauigkeit der Originaldaten bei $98 \%$ entsprechend einer Wolkenverdeckung von $70 \%$. Die Wolken können ebenfalls stark reduziert werden, wobei die Genauigkeit nur geringfügig absinkt. Die Methode ist also sehr wirksam in der Reduktion der Wolkenverdeckung ohne große Genauigkeitseinbußen. Dadurch können die Satellitendaten wesentlich effektiver für wasserwirtschaftliche Zwecke eingesetzt werden. Zwei wasserwirtschaftliche Anwendungen der MODIS Daten werden im Folgenden beschrieben.

\section{Fallstudie - Validierung eines flächendetaillierten Schneemodells}

Hochschwab, Rax, Schneeberg und Schneealpe dienen als Quelleinzugsgebiete für die Wiener Hochquellwasserleitung. Im Rahmen eines Kooperationsprojektes zwischen der TU Wien (als wissenschaftliche Partner) und den Wiener Wasserwerken (MA31) (als Betreiber der Wasserversorgungsanlagen im Untersuchungsgebiet) wurde ein Methode entwickelt, die zeitlich-räumliche Verteilung des Schneewasserwertes zu bestimmen. Die Kenntnis der Schneerücklage ist nicht
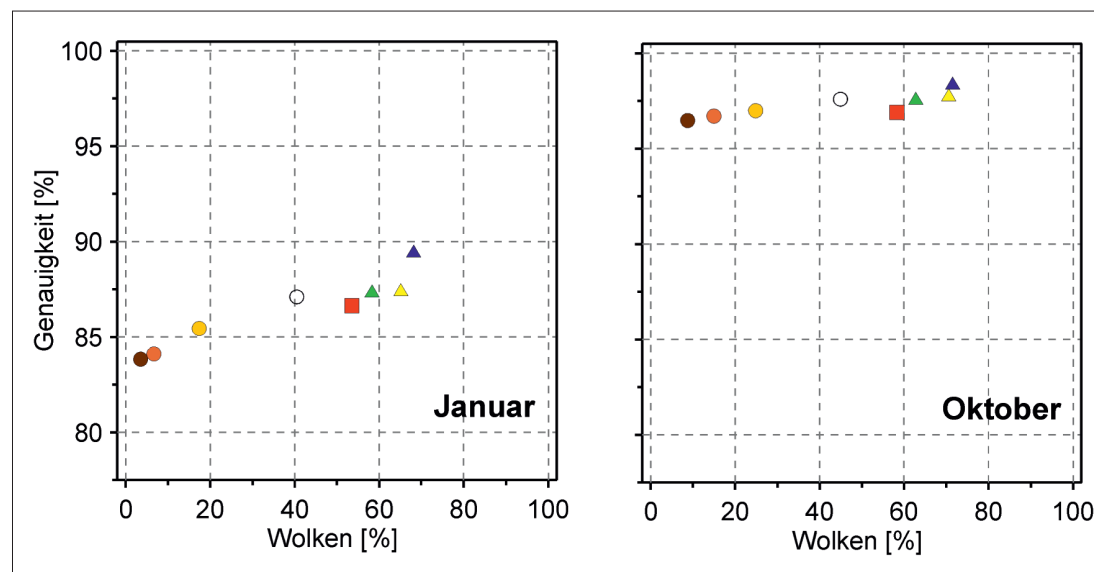
$\triangle$ AQUA
$\triangle$ KOMBINIERT
ZEIT F. [1 Tag]
ZEIT F. [5 Tage]
$\triangle$ TERRA
RAUM F.
ZEIT F. [3 Tage]
- ZEIT F. [7 Tage]

Abb. 4: Genauigkeit der MODIS Schneeklassifizierung (schneebedeckt bzw. aper) im Vergleich zu Messungen der Schneehöhe im Messnetz des Hydrographischen Dienstes in Österreich. Die Symbole zeigen verschiedene Methoden zur Reduktion der Wolkenverdeckung, wodurch allerdings die Genauigkeit etwas reduziert wird. Dreiecke stellen die Aqua bzw. Terra Daten dar, das Quadrat ein räumliches Filter, Kreise zeitliche Filter (aus Parajka und Blöschl 2008a).

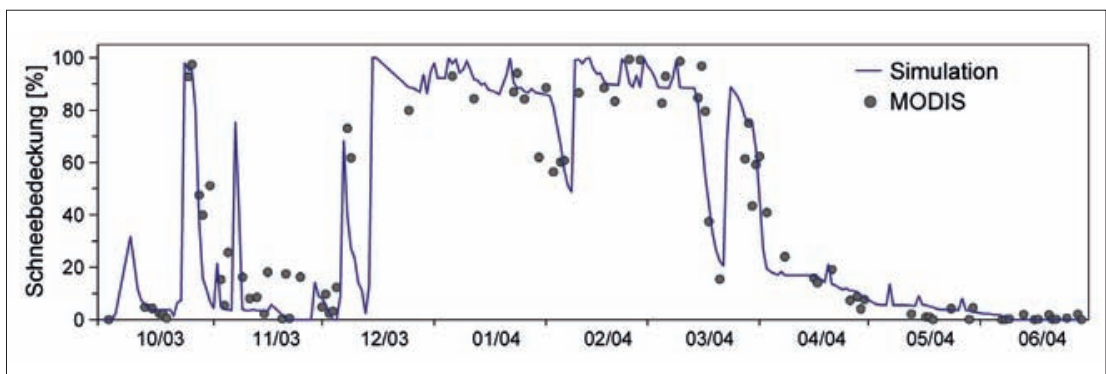

Abb. 5: Schneebedeckte Fläche die durch das Schneemodell simuliert wurde (Linie) im Vergleich zur schneebedeckten Fläche aus MODIS Satellitendaten (Punke) im Hochschwab Gebiet.

nur wichtig zur Abschätzung der zukünftigen Quellschüttungen, sondern auch zur Einschätzung, mit welcher Zeitverzögerung eventuelle Verunreinigungen an den Quellen austreten könnten.

Die Grundidee der Studie war es, alle verfügbaren für die Schneedecke relevanten Informationen zu kombinieren, und mit Hilfe eines Schneedeckenmodells die Verteilung des Schneewasserwertes zu simulieren. Das Schneemodell wurde als prozessorientiertes Rastermodell entsprechend dem Vorbild von Blöschl et al. (1991) formuliert. Die räumliche Modellstruktur besteht aus einem $25 \mathrm{~m}$ Raster, auf dem die Geländeinformation und die Vegetationsinformation (aus Landsat) zur Verfügung steht. Für jedes Rasterelement wird der Schneedeckenaufbau und die Schneeschmelze auf Stundenbasis simuliert. Als Eingangsdaten werden Hydrometeorologische Daten (Niederschlag, Lufttemperatur, Luftfeuchtigkeit, Windgeschwindigkeit, Globalstrahlung) zur Berechung der Energiebilanz und der Schneedeposition verwendet. Schneekurse liefern Daten des Schneewasserwerts, die zur Modellierung der Schneeverfrachtung durch Wind herangezogen wurden. Da es sich sowohl bei den hydrometeorologischen Daten als auch bei den Schneekursen um punktuelle Daten handelt ist es essentiell, flächendeckende Information über die Schneedecke einzubeziehen. Zur Erfassung der kleinräumigen Variabilität der Schneedecke wurden terrestrische digitale Kameras aufgestellt, aus denen die Schneeverteilung rückgerechnet wurde. Zur Erfassung der großräumigen Variabilität der Schneedecke wurden SPOT, ENVISAT und MODIS Satellitendaten herangezogen.

Flächendetaillierte Modelle für den Aufbau und die Ablation der Schneedecke müssen - wie andere hydrologische Modelle - für ein bestimmtes Gebiet geeicht und verifiziert werden, bevor sie für wasserwirtschaftliche Anwendungen eingesetzt werden können. Die Eichung zur Bestimmung optimaler Modellparameter erfolgte in dieser Studie in drei Schritten: 


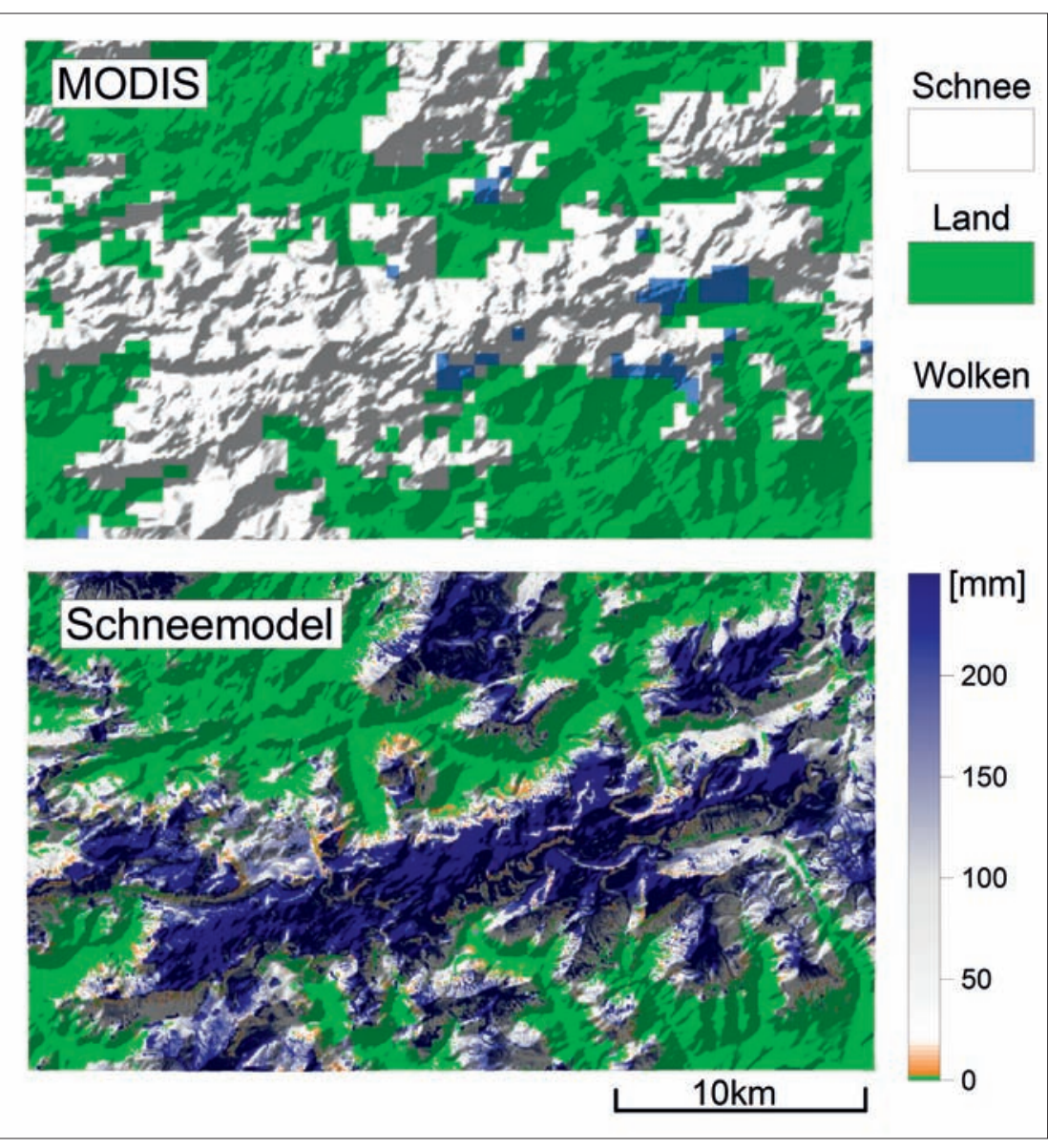

Abb. 6: Schneebedeckung aus MODIS Satellitendaten im 500 m Raster (oben) im Vergleich zum mit dem Schneemodell simulierten Schneewasserwert im 25 m Raster (unten) am 22. März 2003 im Hochschwab Gebiet.

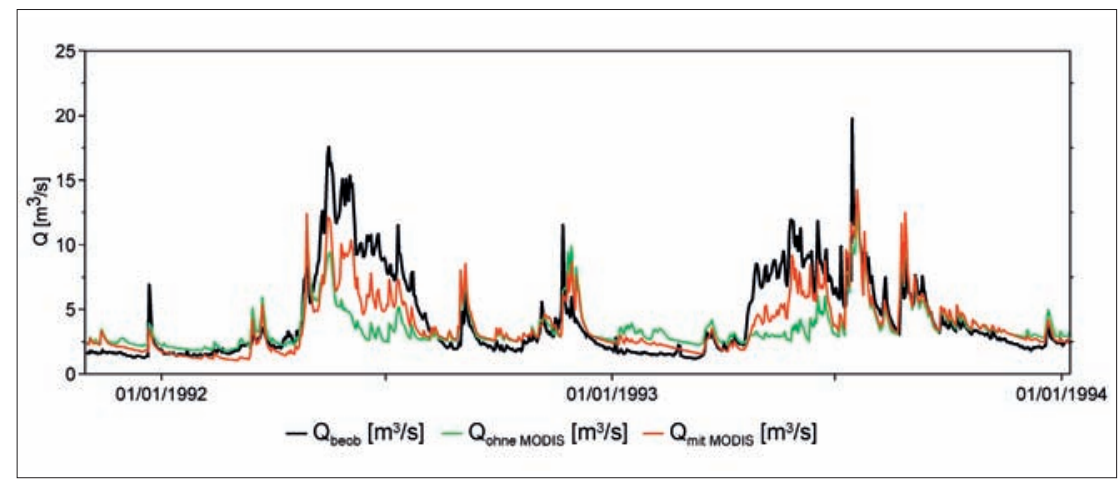

Abb. 7: Beispiel der Abflusssimulation für den Pegel Schruns (102 km²) mit und ohne Verwendung von MODIS Fernerkundungsdaten der Schneebedeckung für einen Verifikationszeitraum.

Parameterwahl auf Basis von Erfahrungswerten; globale Eichung räumlich konstant angesetzter Parameter auf Basis von Schneemustern; und zusätzlich eine flächendetaillierte Eichung auf Basis von Schneemustern, bei der Methoden der vierdimensionalen Daten-Assimilation verwendet wurden. Die Verifikation erfolgte wiederum mit Hilfe von Schneemustern für einen unabhängigen Daten- satz.

Die Verwendung von Fernerkundungsinformation wird hier beispielhaft für den MODIS Sensor dargestellt. Die MODIS Daten wurden in zweierlei Weise verwendet. In einem ersten Schritt wurde die prozentuelle Schneebedeckung für bestimmte Quelleinzugsgebiete und für das Gesamtuntersuchungsgebiet ausgewertet. Dabei wurde die MODIS Schnee- bedeckung verglichen mit der simulierten Schneebedeckung. Dieser Vergleich gibt Hinweise auf eine mögliche systematische Unter- oder Überschätzung des Schneevorrates durch das Modell. Abbildung 5 zeigt einen derartigen Vergleich für die Schneedeckensaison 2003/2004 im Hochschwabgebiet. Um den Einfluss der Bewölkung zu reduzieren, sind die MODIS Daten nur für Tage mit weniger als $60 \%$ Bewölkung dargestellt. Man erkennt, dass das Modell die Schneebedeckung sowohl in der zeitlichen Dynamik als auch im Gesamtniveau der Schneebedeckung sehr gut wiedergibt. Es ist also keine systematische Unter- oder Überschätzung des Schneevorrates durch das Modell zu erwarten. In einem zweiten Schritt wurde die simulierte räumliche Verteilung des Schneewasserwertes verglichen mit den aus MODIS abgeleiteten Schneemustern. Der große Vorteil von Fernerkundungsdaten besteht darin, dass sie flächendeckend zur Verfügung stehen. Man kann zwar nicht den Schneewasserwert ablesen, doch sehr gut die simulierte Schneegrenze mit den Fernerkundungsdaten verifizieren. Im Süden des Untersuchungsgebietes wird der Schneevorrat durch das Modell etwas überschätzt, insgesamt ist aber für das Untersuchungsgebiet eine ausgezeichnete Übereinstimmung von Simulation und Daten festzustellen. Es ist also anzunehmen, dass sich die raum-zeitliche Verteilung des Schneerückhalts und der Schneeschmelze mit dem geeichten und verifizierten Modell zuverlässiger bestimmen lässt, als dies ohne Vergleiche mit beobachteten Schneemustern möglich ist. Eine Einbindung des Modells in ein operationelles Vorhersagesystem ist in Arbeit.

\section{Fallstudie - Eichung eines regionalen Niederschlag- Abflussmodells}

In der zweiten Fallstudie geht es um die flächendeckende Modellierung der Wasserbilanz für Österreich, die im Rahmen zweier Europäischer Projekte (AWARE, M.CURIE) durchgeführt wurde Parajka et al. (2005ab, 2007ab). Die Schwierigkeit be einer derartigen Wasserbilanzrechnung besteht unter anderem darin, dass die Niederschlagsstationen vor allem in den Tallagen angeordnet sind und die Hochlagen vergleichsweise wenige Stationen besitzen. Außerdem wird, gerade im Gebirge, der Niederschlag durch die Messung systematisch unterschätzt. Aus beiden Grün- 

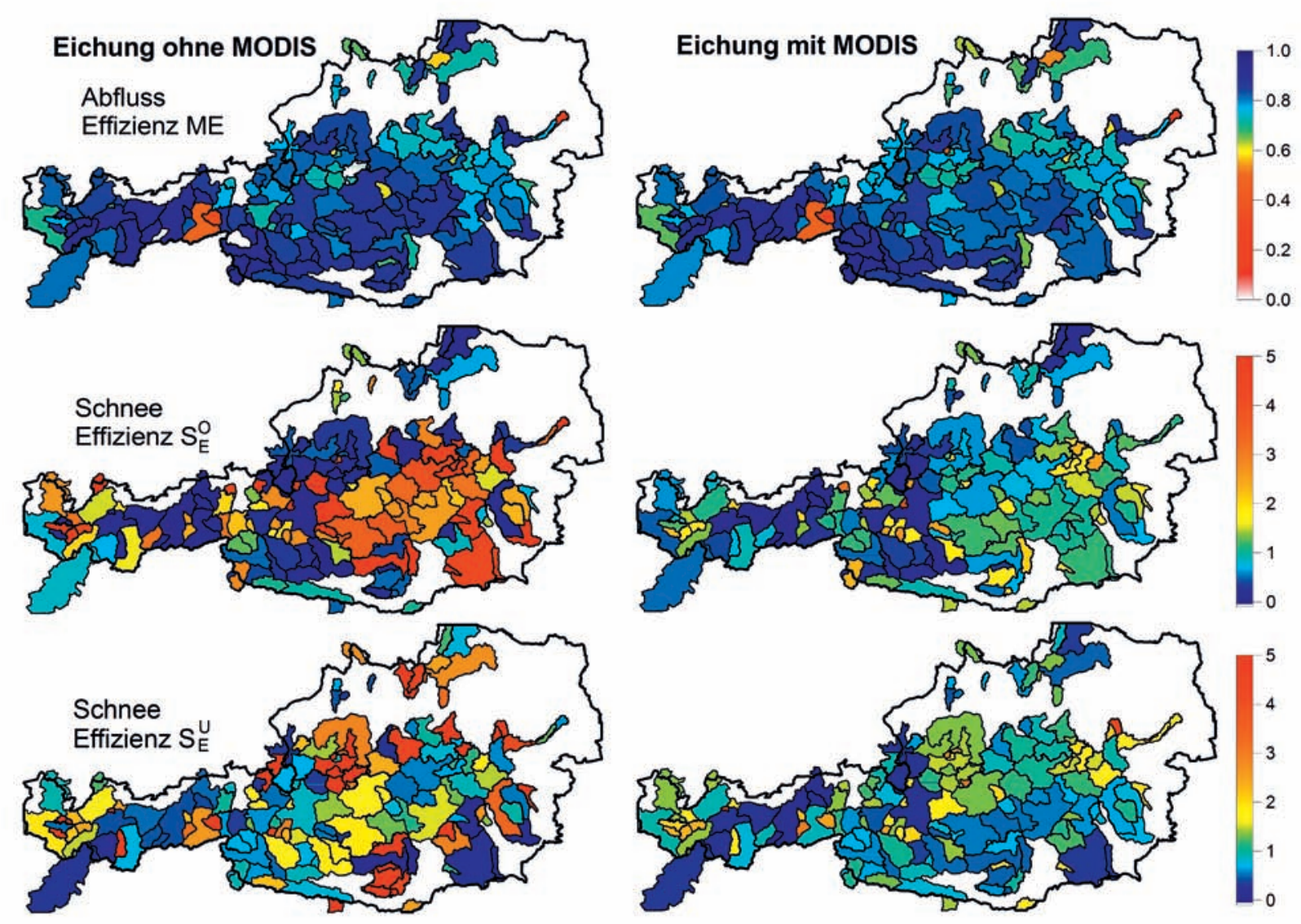

Abb. 8: Effizienz der Simulation von Tageswerten des Abflusses für die Varianten mit und ohne Verwendung von MODIS (oben). Fehler der Schneesimulation mit und ohne Verwendung von MODIS (Mitte und unten). Zeitraum 2003-2005. In allen Bildern entspricht die Farbe rot schlechten Simulationen, dunkelblau guten Simulationen.

den ist der mit hydrologischen Modellen simulierte Schneewasserwert im Gebirge wesentlich unsicherer als im Flachland. Um zuverlässigere Aussagen über die Wasserbilanz $\mathrm{zu}$ erhalten werden auch hier Fernerkundungsdaten der Schneebedeckung herangezogen.

Es bestehen zwei Möglichkeiten, Schneedaten aus der Fernerkundung in die Modellierung einzubeziehen. Die erste Möglichkeit besteht darin, die simulierten Schneewasserwerte in Echtzeit mittels der Schneedaten zu aktualisieren wobei hier Methoden der Datenassimilation wie etwa Ensemble Kalman Filtering (Blöschl et al. 2008; Komma et al. 2008) zur Anwendung kommen. Die zweite Möglichkeit besteht darin, die Schneedaten bei der Modellidentifikation bzw. Eichung zu verwenden, um zuverlässigere Modellparameter zu erhalten, als dies ohne Fernerkundungsdaten möglich ist. Dabei spielt auch die Datenkonsistenzprüfung eine große Rolle, die durch Fernerkundungsdaten erleichtert wird.
In der hier beschriebenen Fallstudie (Parajka und Blöschl 2008b) wird die Schneeinformation in die Zielfunktion bei der Modelleichung einbezogen. Die Zielfunktion $Z$ besteht aus zwei Teilen, einem Teil $Z_{Q}$, der die Abweichung zwischen simuliertem und beobachtetem Abfluss beschreibt, und einen Teil $Z_{S}$, der die Abweichung zwischen simulierter und beobachteter Schneebedeckung beschreibt:

$Z=w_{S} \cdot Z_{S}+\left(1-w_{S}\right) \cdot Z_{Q}$

$Z_{Q}$ wurde dabei auf Basis der Nash-Sutcliffe Effizienz gewählt, $Z_{S}$ als Summe der Fehler der Überschätzung $S_{E}^{O}$ und der Unterschätzung $S_{E}^{U}$. Der Unterschätzungsfehler zählt die Anzahl der Tage, an denen das hydrologische Modell keinen Schnee simuliert, aber die MODIS Daten eine Schneebedeckung größer als ein Schwellenwert angeben. Der Überschätzungsfehlers ist analog definiert. Das Gewicht $w_{s}$ wurde nach Sensitivitätsanalysen auf 0,9 gesetzt. Zum Vergleich wurden auch Varianten simuliert, bei denen die MODIS Daten nicht in die Eichung des hydrologischen Modells einflossen $\left(w_{S}=0\right)$.

Ein Beispiel für den Vergleich der beiden Varianten (mit und ohne Verwendung von MODIS Fernerkundungsdaten der Schneebedeckung) für den Pege Schruns ist in Abbildung 7 gezeigt. Wie man sieht verbessert die Schneeinformation auch die Abflusssimulation wesentlich. Es handelt sich dabei um einen Verifikationszeitraum, der nicht für die Eichung herangezogen wurde. Eine derartige Verbesserung der Abflusssimulation kann freilich nicht für alle Einzugsgebiete erwartet werden, insbesondere nicht für Gebiete, in denen die Abflusssimulationen genauer den Beobachtungen entsprechen als im gezeigten Beispiel. Eine flächige Auswertung ist deshalb in Abbildung 8 für 148 österreichische Gebiete dargestellt. Die linken Karten in Abbildung 8 zeigen die Modellgüte ohne Verwendung von MODIS Daten. Die Abflusseffizienz ME liegt um 
0,8 oder höher, das ist ein für regionale $\mathrm{Si}$ mulationen durchaus zufriedenstellender Wert. Eine Nash-Sutcliffe Effizienz von $\mathrm{ME}=1$ bedeutet perfekte Simulation, $\mathrm{ME}=0$ entspricht dem Mittelwert. Ohne Verwendung von MODIS Daten wird die Schneebedeckung durch das Modell häufig deut lich überschätzt (rote Farben bei $S_{E}^{O}$ ) aber auch eine Unterschätzung tritt häufig auf. Die rechten Karten in Abbildung 8 zeigen die Modellgüte mit Verwendung von MODIS Daten. Die Modellgüte der Abflusssimulation ändert sich kaum, die Modellgüte der Schneesimulation wird wesentlich verbessert. Während sich also im Mittel die Güte der Abflusssimulationen kaum ändert, ist eine Verbesserung vor allem in $\mathrm{Si}$ tuationen zu erwarten, die spezielle hydrologische Verhältnisse aufweisen. Reszler et al. (2008) zeigte, dass für spezielle Verhältnisse wie Regen auf Schnee und sehr mächtige Schneedecken eine gute Modellstruktur und zutreffende Modellparameter für gute Abflussprognosen besonders wichtig sind. Parajka und Blöschl (2008b) zeigten auch, dass der Gewinn der Verwendung von Fernerkundungsdaten der Schneebedeckung für die hydrologische Modellierung besonders hoch ist, wenn die Anzahl der Niederschlagsstationen gering ist. Das macht die Fernerkundung des Schneevorrates besonders für die alpinen Teile Österreichs besonders attraktiv.

\section{Abschließende Bemerkungen}

Schnee lässt sich mit Fernerkundung zwar nur zum Teil erfassen, aber die Schneebedeckung und auch die Änderung der Schneefeuchte bieten bereits genügend Information, um eine qualifizierte Analyse der Schneevorrats durchführen zu können. Die Bewölkung behindert optische Methoden der Fernerkundung, doch existieren Methoden, mit denen die Wolkenverdeckung wesentlich reduziert werden kann, ohne die Genauigkeit der Schneebedeckungsklassifikation merklich zu verringern. Anhand zweier Fallbeispiele - der Validierung eines flächendetaillierten Schneemodells und der regionalen Wasserbilanzmodellierung wurde in dieser Arbeit gezeigt, dass die Schneeinformation eine wesentliche Verbesserung der hydrologischen Simulationen und eine verbesserte Einschätzung deren Zuverlässigkeit gegenüber den herkömmlichen Punktdaten von Niederschlag, Lufttemperatur etc. und Abflussdaten alleine ermöglicht. Fernerkundungsdaten der Schneedecke sind deshalb sowohl für wasserwirtschaftliche Simulationen als auch für Echtzeitprognose sehr interessant. Bei der Auswahl der Sensoren für operationelle hydrologische Anwendungen ist auf die folgenden Aspekte zu achten:
- Räumliche und zeitliche Auflösung

- Genauigkeit

- Datenausfal

- Termingerechte Verfügbarkeit und

- Kosten

Unter diesen Gesichtspunkten kommen für österreichische Verhältnisse vor allem optische Sensoren in Frage, wie etwa das Moderate Resolution Imaging Spectroradiometer (MODIS).

\section{Danksagung}

Die Autoren danken dem FWF (P18993N10), derEU(AWARE, SST4-CT-2004-012257 und KATER II Interreg IIIB CADSES) sowie den Wiener Wasserwerken (MA31) für die Förderung der in diesem Artikel beschriebenen Untersuchungen sowie dem Hydrographischen Zentralbüro (HZB) für die Bereitstellung von Daten.

\section{Korrespondenz:}

Dr. Jurpa Parajka, Univ.Prof. Dr. Günter Blösch

Institut für Wasserbau und Ingenieurhydrologie

Technische Universität Wien

Karlsplatz 13/222, 1040 Wien

E-ail: parajka@hydro.tuwien.ac.at

Univ. Prof. Dr. Josef Jansa

Institut für Photogrammetrie und Fernerkundung

Technische Universität Wien

Gußhausstraße 27-29, 1040 Vienn

E-Mail: ij@ipf.tuwien.ac.at

\section{LITERATUR}

Blöschl G, Reszler C, Komma J (2008) A spatially distributed flash flood forecasting model. Environmental Modelling \& Software, 23 (4), pp. vironmentain.

464-478.

Blöschl G, Kirnbauer R, Gutknecht D
(1991) Distributed snowmelt simulations in an alpine catchment. 1. Model evaluation on the basi of snow cover patterns. Water Resources Research, 27 (12), pp. 3171-3179.

Blöschl G, Kirnbauer R, Jansa J, Kraus K, Kuschnig G, GutknechtD, Reszler Ch (2002) Einsatz von Fernerkundungsmethoden zur Eichung und Verifikation eines flächendetaillierten Schneemodells. Österreichische Wasser- und Abfallwirtschaft, 54 (1/2), pp. 1-16.

fallwirtschaft, 54 (1/2), pp. 1-16. Deems JS, Painter TH (2006) LIDAR measurement of snow depth: accuracy and error sources. In: Proceeding of International Snow Science Workshop, Telluride CO, Oktober 2006. Derksen C, Walker A, Goodison B (2005) Evaluation of passive microwave snow water equivalent retrievals across the boreal forest/tundra transition of western Canada. Remote Sensing of Environment, 96 (3-4), 315-327.

Dozier J (1989) Spectral signature of Alpine snow cover from the Landsat Thematic Mapper. Remote Sensing of Environment, 28:9-22. ESA (2009) http://www.esa.int

ESA (2009) http://www.esa.int Casey K, Chien JYL, DiGirolamo NE, Klein AG,
Powell HW, Tait AB (2001) Algorithm theoretical basis document (ATBD) for the MODIS snow and sea ice-mapping algorithms, available at: $h$ ttp:// modis-snow-ice.gsfc.nasa.gov/atbd01.html. modis-snow-ice.gsfc.nasa.gov/atbd01.html. Komma J, Blöschl G, Reszler C (2008) Soil
moisture updating by Ensemble Kalman Filtering in real-time flood forecasting. Journal of Hydrology, 357, pp. 228-242.

Landsat (2009) Landsat Program. http://landsat. gsfc.nasa.gov/

MODIS (2009) http://modis.gsfc.nasa.gov/ Nagler Th, Rott H (2000) Retrieval of wet snow by means of multitemporal SAR data. IEEE Transactions on Geoscience and Remote Sensing, 38(2):754-765.

Parajka J, Blöschl G (2006) Validation of MODIS Parajka J, Blöschl G (2006) Validation of MODIS Snow cover images over Austria. Hydrology Earth System Sciences, 10, pp. 679-689.
Parajka J, Blöschl G (2008a) Spatio-temporal combination of MODIS images - potential for snow cover mapping. Water Resources Research, 44, article number W03406. Parajka J, Blöschl G (2008b) The value of MODIS snow cover data in validating and calibrating conceptual hydrologic models, Journal of Hydrology, 358, 240-258.

Parajka J, Blöschl G, Merz R (2007a) Regional calibration of catchment models: Potential for ungauged catchments. Water Resources Research, 43 , article number W06406.
Parajka J, Merz R, Blöschl G (2005a) A comparison of regionalisation methods for catchment model parameters. Hydrology and Earth Systems model parameters. Hydrol

Parajka J, Merz R, Blöschl G (2005b) Regionale Parajka J, Merz R, Blöschl G (2005b) Regionale
Wasserbilanzkomponenten für Österreich auf Tagesbasis Österreichische Wasser- und Abfallwirtschaft, 57 (3/4), pp. 43-56.

Parajka J, Merz R, Blöschl G (2007b) Uncertainty and multiple objective calibration in regional water balance modeling - Case study in 320 Austrian catchments. Hydrological Processes, 21 pp. 435-446.

Reszler Ch, Komma J, Blöschl G, Gutknecht D (2008) Dominante Prozesse und Ereignistypen zur Plausibilisierung flächendetaillierter Niederschlag-Abflussmodelle. Hydrologie und Wasserbewirtschaftung, 52 (3), pp. 120-131. Riggs GA, Hall DK, Salomonson VV (1994) A snow index for the Landsat Thematic Mapper and Moderate Resolution Imaging Spectroradiometer. In: Proceeding of IEEE Geoscience and Remote Sensing Conference 1994, Vol.4, pp. 1942-1944.

Spot (2009) SPOT Image. http://www.spotimage. $\mathrm{fr} /$

Ulaby FT, Moore RK, Fung AK (1986) Microwave remote sensing: active and passive. Volume III: From theory to applications. Artech House. 